\title{
In-Situ TEM Observations of Interface Sliding and Migration in a Refined Lamellar TiAl Alloy
}

\author{
L. M. Hsiung, A. J. Schwartz, T.G. Nieh
}

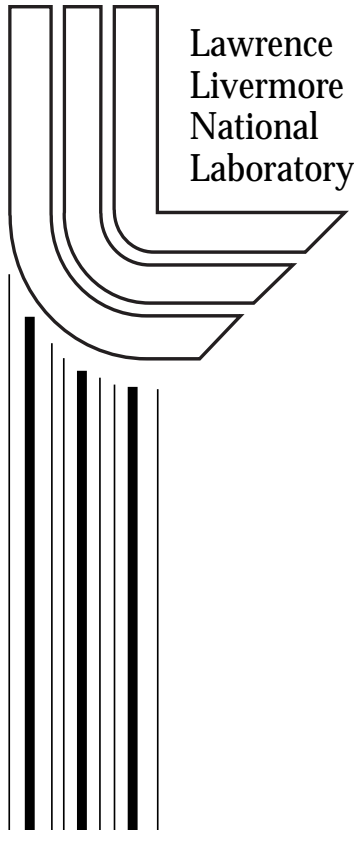

February 18, 2004 
This document was prepared as an account of work sponsored by an agency of the United States Government. Neither the United States Government nor the University of California nor any of their employees, makes any warranty, express or implied, or assumes any legal liability or responsibility for the accuracy, completeness, or usefulness of any information, apparatus, product, or process disclosed, or represents that its use would not infringe privately owned rights. Reference herein to any specific commercial product, process, or service by trade name, trademark, manufacturer, or otherwise, does not necessarily constitute or imply its endorsement, recommendation, or favoring by the United States Government or the University of California. The views and opinions of authors expressed herein do not necessarily state or reflect those of the United States Government or the University of California, and shall not be used for advertising or product endorsement purposes. 


\title{
IN-SITU TEM OBSERVATIONS OF INTERFACE SLIDING AND MIGRATION IN A REFINED LAMELLAR TIAI ALLOY
}

\author{
L.M. Hsiung ${ }^{*}$, A.J. Schwartz, and T.G. Nieh \\ University of California, Lawrence Livermore National Laboratory, \\ L-352, P.O. Box 808 \\ Livermore, CA 94551-9900, USA.
}

\begin{abstract}
The stability of lamellar interfaces in lamellar TiAl by straining at ambient temperatures has been investigated using in-situ straining techniques performed in a transmission electron microscope in order to obtain direct evidence to support the previously proposed creep mechanisms in refined lamellar TiAl based upon the interface sliding in association with the cooperative motion of interfacial dislocations. The results have revealed that both sliding and migration of lamellar interfaces can take place as a result of the cooperative motion of interfacial dislocations.
\end{abstract}

Keywords: A. Transmission electron microscopy, in-situ straining; B. Lamellar TiAl, interface dislocation, interface sliding, interface migration

*) Corresponding author: Phone: 925424 3125, Fax: 925424 3815, e-mail: hsiung1@llnl.gov

\section{Introduction}

It has been reported previously that the mobility of interfacial dislocations can play a crucial role in the creep deformation behavior of refined lamellar TiAl $[1,2]$. Since the operation of lattice dislocations within refined $\alpha_{2}$ and $\gamma$ lamellae is largely restricted, the motion of interfacial dislocations becomes the major strain carrier for plasticity. Results of ex-situ TEM investigation have revealed the occurrence of interface sliding in low-stress (LS) creep regime and deformation twinning in high-stress $(H S)$ creep regime. These results have led us to propose that interface sliding associated with a viscous glide of pre-existing interfacial dislocations is the predominant creep mechanism in $L S$ regime and interfaceactivated deformation twinning in $\gamma$ lamellae is the predominant creep mechanism in $H S$ regime [1,2]. Stress concentration resulted from the pileup of interfacial dislocations is suggested to be the cause for the interface-activated deformation twinning. Accordingly, the creep resistance of refined lamellar TiAl is considered to depend greatly on the cooperative motion of interfacial dislocations, which in turn may solely be controlled and hindered by the interfacial segregation of solute atoms (such as $W$ ) or interfacial precipitation [3]. Furthermore, through the in-situ TEM investigation, we also found that the lamellar interfaces could migrate directly through the cooperative motion of interfacial dislocations. That is, the $\gamma / \gamma$ and $\gamma / \alpha_{2}$ interfaces can migrate through interface sliding and lead to the coalescence or shrinkage of constituent lamellae (i.e. microstructural instability), which results in a weakening effect when refined lamellar TiAl is employed for engineering applications. Although it is anticipated that interface sliding and migration are prevalent at elevated 
temperatures, the present in-situ straining study reveals the instability of lamellar interfaces at ambient temperatures.

\section{Experimental}

A fully-lamellar (FL) TiAl alloy with a nominal composition of Ti-47A1-2Cr-1Nb-1Ta (at.\%) was fabricated by a powder metallurgy process, which involves a hot-extrusion of gas-atomized titanium aluminide powder (particle size: - 200 mesh) canned in molybdenum billets and were subsequently hot-extruded at $1400^{\circ} \mathrm{C}$. After extrusion, the rod-shape alloy was stress-relieved at $900^{\circ} \mathrm{C}$ for $2 \mathrm{~h}$ in a vacuum of $\sim 10^{-4} \mathrm{~Pa}$. TEM foils were prepared by twin-jet electropolishing in a solution of $60 \%$ methanol, $35 \%$ butyl alcohol and $5 \%$ perchloric acid at $15 \mathrm{~V}$ and $-30^{\circ} \mathrm{C}$. Interfacial substructures were examined using JEOL200CX transmission electron microscope. Dislocation structure and the core structure of interfacial dislocations were also examined using weak-beam dark field (WBDF) and highresolution (HRTEM) imaging techniques. To investigate strain-induced interface migration, an in-situ straining experiment was performed at room temperature in a JEOL-200CX transmission electron microscope using a single-tilt straining holder. A gear-drive translation mechanism was activated through the foot pedals with the deformation rate controlled by the speed of y-axis tilt. Plastic deformation took place and was recorded right after a short period of time in which elastic deformation was first distinguished from the motion of bend extinction contours. A hi- 8 videocassette recorder attached to a TV rate camera in the microscope was used to record the straining events (e.g., dislocation motion/interaction, interface migration). After completing the experiment, video images of special interest were printed out for analysis. An in-situ TEM experiment was also conducted by electron-beam heating of a creep-deformed sample $\left(138 \mathrm{MPa}, 760^{\circ} \mathrm{C}\right)$ in order to investigate the thermal effect on the stability of interfacial dislocations.

\section{Results and Discussion}

\subsection{Interfacial substructures}

There are in general two types of lamellar interfaces within FL-TiAl [4-6], i.e. (1) the $\gamma / \alpha_{2}$ interphase interface which has an orientation relationship: (0001) $)_{\alpha 2} \|(111)_{\gamma}$ and $\langle 11 \overline{2} 0\rangle_{\alpha 2} \|\langle 1 \overline{1} 0\rangle_{\gamma}$, and (2) the $\gamma / \gamma$ interfaces which include true twin, pseudo twin, and order-fault interfaces. Types (2) interface is also referred as twin-related interfaces hereafter. A typical TEM observation of lamellar interfaces viewed from the [011] $]_{\gamma}$ edge-on orientation is shown in Fig. 1. Notice that interfacial dislocations (IDs hereafter) can be found in both $\gamma / \gamma$ and $\gamma / \alpha_{2}$ interfaces. Figure 1(b) was imaged with a tilting angle of $\sim 15^{\circ}$ from Fig. 1(a). Several dislocation tips (appeared as white dots) in Fig. 1(a), and the corresponding dislocation lines appeared in Fig. 1(b) are marked by arrows. In general, the dislocation density in $\gamma / \alpha_{2}$ interphase interfaces is higher than that in $\gamma / \gamma$ twin-related interfaces as a result of a greater lattice and thermal misfit between $\gamma$ and $\alpha_{2}$ lamellae. The core of each ID in $\gamma / \alpha_{2}$ interface contains a small step (ledge) with the step height two layer thickness of the $(111)_{\gamma}$ plane $\left(d_{111}=0.232 \mathrm{~nm}\right)$ is shown in Fig. 2(b), which is consistent with the observations reported elsewhere in literature [7,8]. Accordingly, if an ID moves along the interface, the step moves along with the dislocation and thus, the interface is displaced (advanced) perpendicular to its plane, i.e. the interface will migrate by the distance of a step-height. Therefore, the cooperative motion of IDs will always cause a combination of interface sliding and interface migration as 
illustrated schematically in Fig. 2(c). Since the Burgers vector $(\mathbf{b}=1 / 6<\overline{1} 12>$ ) of IDs is parallel to the (111) $\gamma$ plane, the dislocations need only to glide to cause the interface to slide. Similar properties are also true for $\gamma / \gamma$ interfaces, and a small step is associated with the core of each ID $(\mathbf{b}=1 / 6[1 \overline{1} 2])$ in $\gamma / \gamma$ interface with the step height one layer spacing of the (111) $\gamma$ plane [Fig. 3(a)]. The IDs in $\gamma / \gamma_{\mathrm{T}}$ true-twin interface compensate a small angle departure of the $\gamma / \gamma_{\mathrm{T}}$ interface plane from the exact (111) twin plane, which is schematically illustrated in Fig. 3 (a). The inclination angle $(\theta)$ of the interface plane to the exact twin plane is therefore given by

$$
\tan \theta=\frac{h}{s}
$$

where $\boldsymbol{h}$ is the step height, and $s$ is the average spacing of IDs. Accordingly, as illustrated in Figs. 2(b) and 3(b), it is anticipated that the cooperative motion of interfacial (Shockley partial) dislocations can result in the migration of lamellar interfaces, and thereby lead to the coalescence/shrinkage of the constituent lamellae. The migration rate $\left(v_{i}\right)$ of an interface can be expressed as

$$
v_{i}=\rho v_{d} h
$$

where $\rho$ is the dislocation density, $\boldsymbol{v}_{\boldsymbol{d}}$ the dislocation velocity, and $\boldsymbol{h}$ the step height.

Indirect evidence of the motion of IDs in a $\gamma / \gamma_{T}$ true-twin interface and a $\gamma / \alpha_{2}$ interphase interface are shown in Figs. 4 (a) and (b) respectively, which were observed from a creepdeformed alloy sample (creep stress: $138 \mathrm{MPa}$, creep strain: $0.5 \%$, test temperature: $760^{\circ} \mathrm{C}$ ). Here in Fig. 4(a), several IDs were found to pile up at site $\mathrm{P}$ as a result of the impingement of lattice dislocations to the interface. Figure 4 (b) shows the appearance of fringe contrast trailing along single-array ID on $\gamma / \alpha_{2}$ interphase. These fringe contrasts reveal the formation of a stacking fault ribbon on the wake of each $I D$. The fringe contrast is best viewed when $2 \pi g \cdot R=2 / 3$ or $4 / 3$, where $g$ is the reflection vector and $R(=1 / 3[111])$ is the displacement vector of stacking fault in $\gamma$ lamella. The wavy appearance of those dislocation lines suggests that they were severely dragged during motion, presumably by lattice imperfections such as solute atoms.
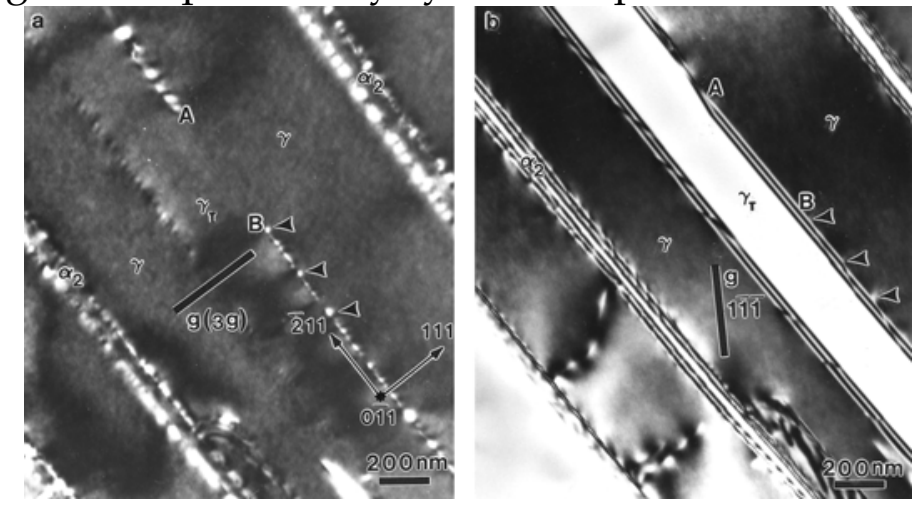

Fig. 1 (a) A WBDF TEM image showing a typical edge-on lamellar structure consisting of $\gamma, \gamma_{\mathrm{T}}$ and $\alpha_{2}$ lamellae within an as-fabricated alloy sample, $Z$ (zone axis) $=[0 \overline{1} 1]_{\gamma}$. (b) A bright-field TEM image showing the existence of interfacial dislocations in both $\gamma / \alpha_{2}$ and $\gamma / \gamma_{\mathrm{T}}$ interfaces, $\mathrm{Z}=[1 \overline{2} 1]_{\gamma}$.

Approved for public release; further dissemination unlimited 


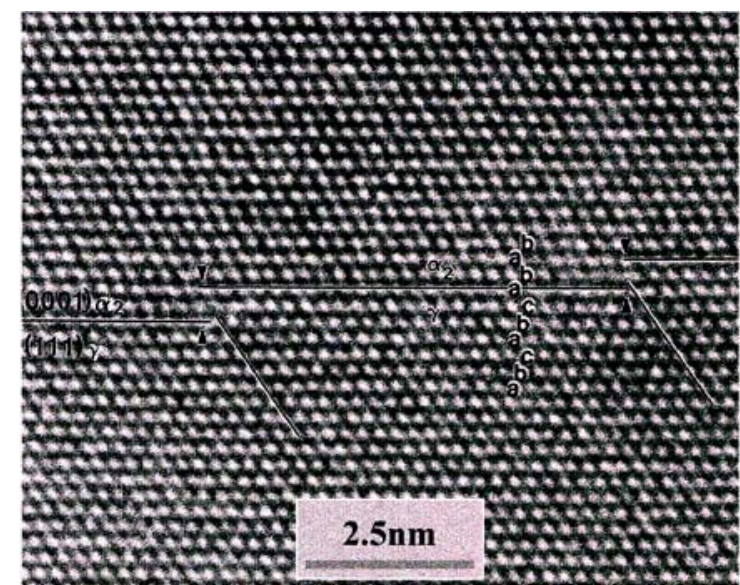

(a)

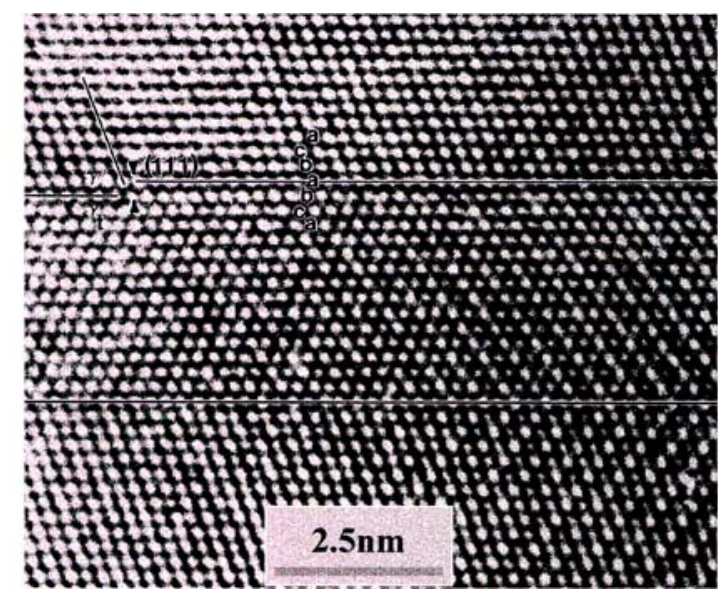

(b)

Fig. 2. HRTEM images showing the core structure of interfacial dislocations in (a) $\gamma / \alpha_{2}$ and (b) $\gamma / \gamma_{\mathrm{T}}$ interfaces. The letters abab and abcabc stand for the stacking sequence of $\alpha_{2}$ and $\gamma$ lamellae, respectively.

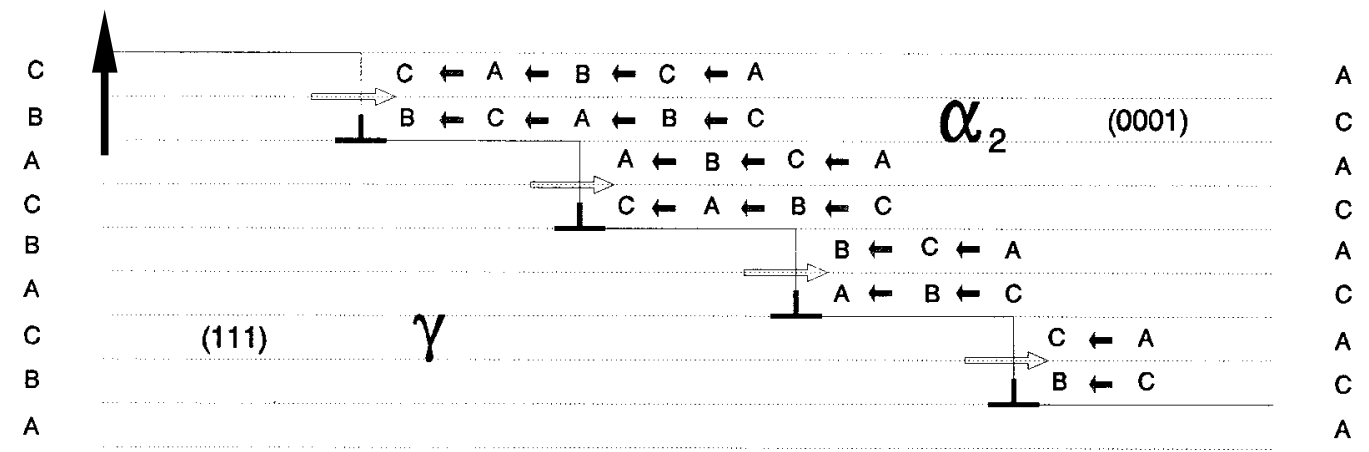

(a)

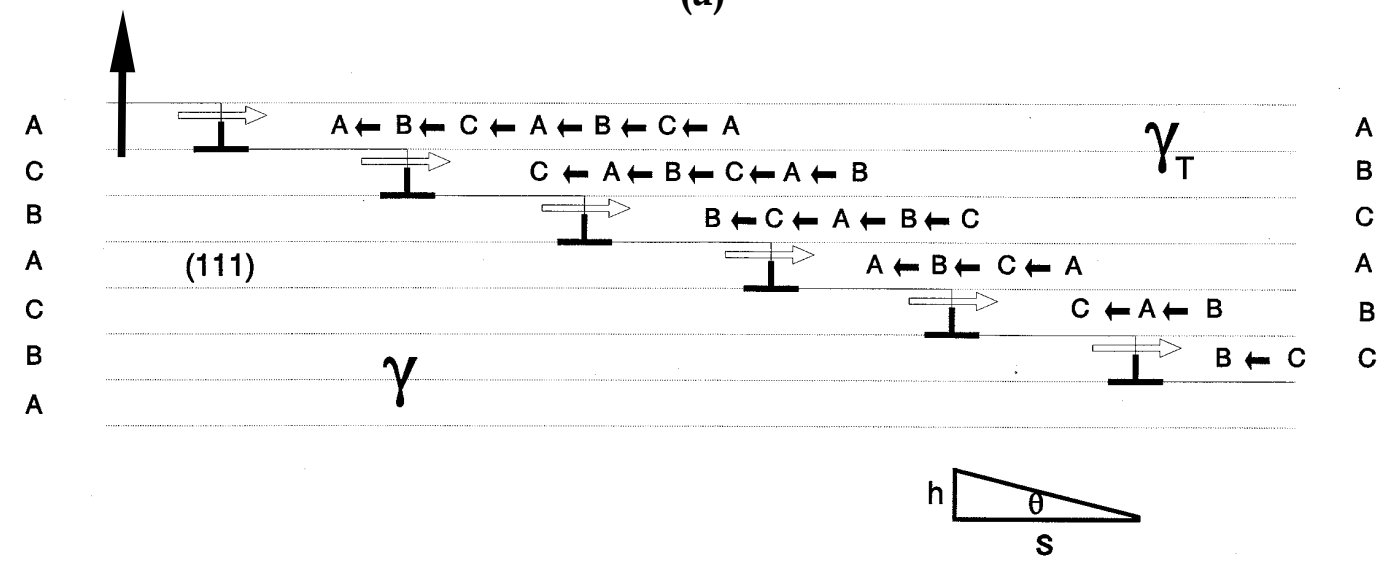

(b)

Fig. 3. Schematic illustrations of (a) an array of interfacial (Shockley partial) dislocations forming a glissile $\alpha_{2} / \gamma$ interface and (b) an array of interfacial (Shockley partial) dislocations in a glissile $\gamma / \gamma_{\mathrm{T}}$ interface. Both interfaces can be migrated by the cooperative motion of the interfacial dislocations. The letters ACAC and ABC stand for the stacking sequence of $\alpha_{2}$-lamella and $\gamma$-lamella, respectively. The direction of interface migration is indicated by $\uparrow$. 


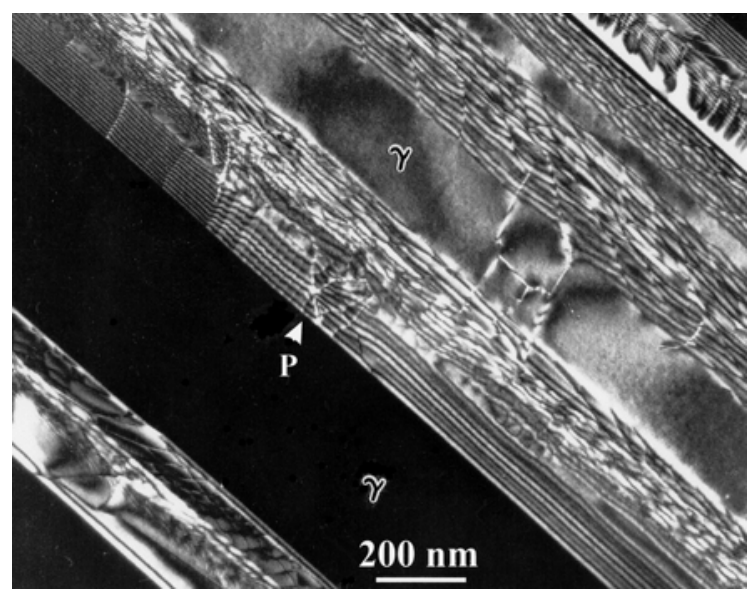

(a)

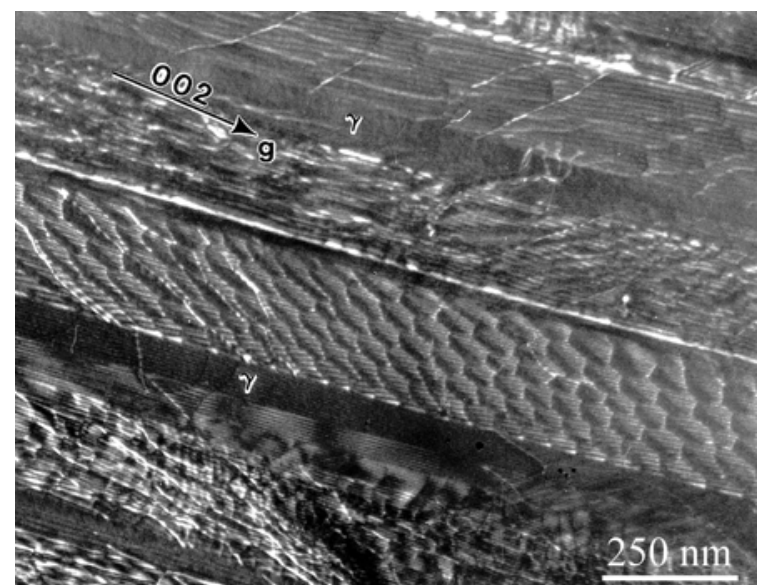

(b)

Fig. 4. WBDF TEM images showing (a) the pileup of an ID array in a $\gamma / \gamma_{\mathrm{T}}$ interface as a result of the impingement of lattice dislocations at site $\mathrm{P}$, and (b) the appearance of fringe contrast of stacking fault trailing along each gliding ID in a $\gamma / \alpha_{2}$ interface. Notice the wavy appearance of the dislocation array presumably caused by the dragging of solute atoms.

\subsection{In-situ observation of interface sliding}

A direct observation of interface sliding and migration has been obtained from an in-situ straining experiment. Throughout the in-situ straining experiment (conducted at room temperature), the motion of IDs was mainly observed in $\gamma / \gamma$ twin-related interfaces. This indicates that the mobility of IDs in $\gamma / \alpha_{2}$ interface is much lower than that of IDs in $\gamma / \gamma$ twin-related interfaces at room temperature. Typical video images recorded from the insitu straining experiment for the motion of IDs in a pair of edge-on twin interfaces are demonstrated in Figs. 5 (a) and (b), in which the identical IDs are marked by arrows. It is seen that dislocations in the left interfaces moved cooperatively about $17 \mathrm{~nm}$ downward after 11 seconds, while those in the right interfaces moved cooperatively about an equal distance upward. The average dislocation velocity $\left(v_{d}\right)$ is estimated to be $1.5 \mathrm{~nm} / \mathrm{s}$. With a known dislocation density at the interface $\left(\rho \sim 0.033 \mathrm{~nm}^{-1}\right)$, the interface migration rate $\left(v_{i}\right)$ can be evaluated [according to eqn. (2)] to be $0.012 \mathrm{~nm} / \mathrm{s}$, which is so slow that the interface migration is not detectable within a short period of time.

Results of an in-situ observation of ID motion in a creep-deformed sample under a beam heating condition are presented in Fig. 6. Since the purpose the study is solely to demonstrate the direct observation of ID motion, the amount of temperature increase by beam heating is not a major concern here. It is also noted that a local heating was achieved by focusing the spot size of electron-beam to several microns. Figure 6(a) shows the cooperative motion of a dislocation array (eight interfacial dislocations as indicated) on a $\gamma / \gamma$ interface. The dislocations moved cooperatively about $250 \mathrm{~nm}$ after beam heating for 20 seconds. 

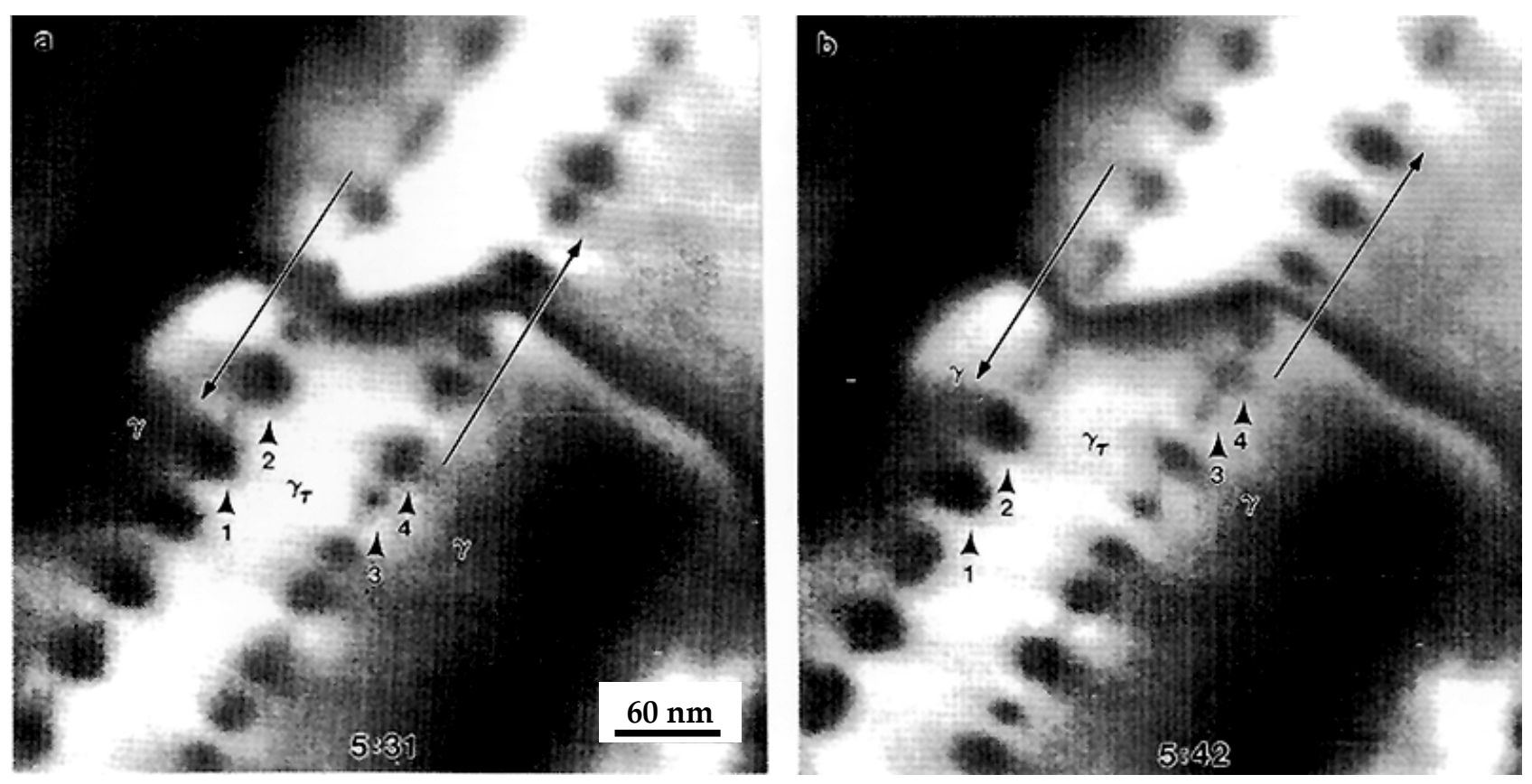

Fig. 5. Two consecutive in-situ video images showing the cooperative motion of interfacial dislocation tips (appeared as black dots) in a pair of $\gamma / \gamma$ interfaces; the moving directions are labeled by long arrows.

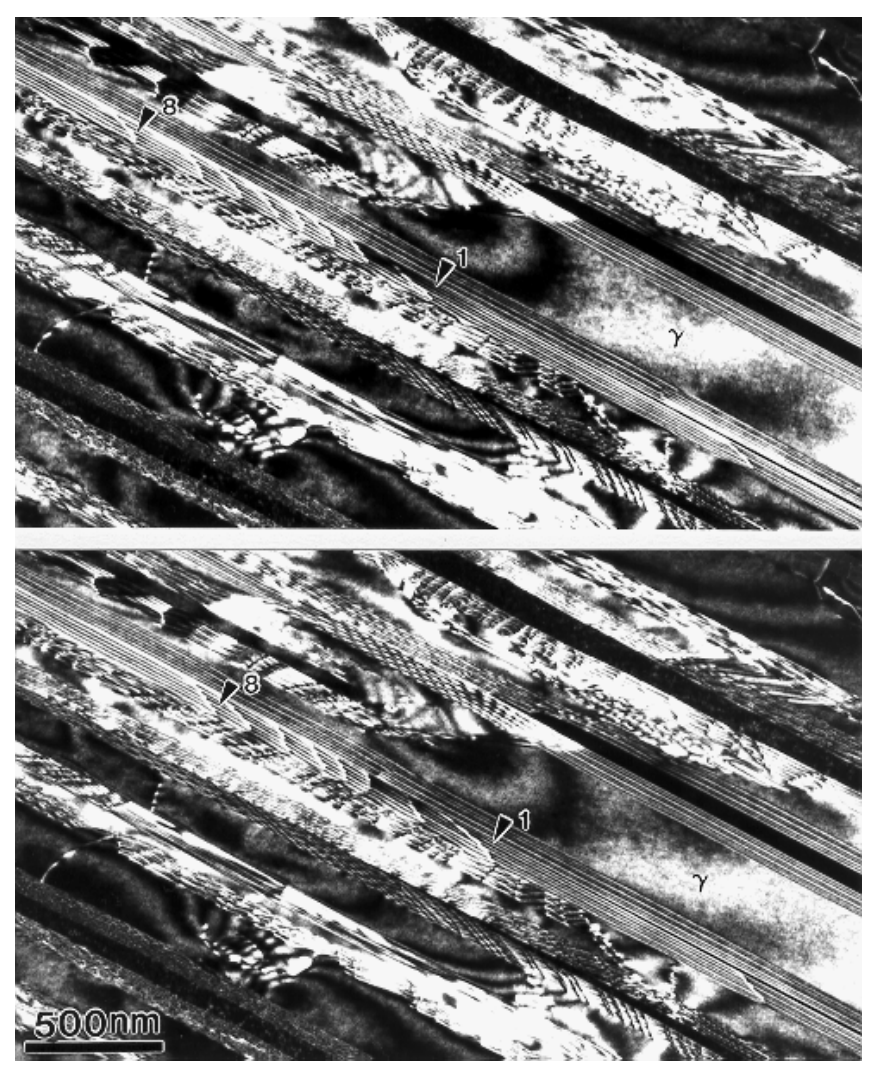

Fig. 6. In-situ TEM images showing the cooperative motion of interfacial dislocation in a $\gamma / \gamma$ interface driven by beam heating (time lapse for beam heating: 20 seconds). 


\subsection{In-situ observation of interface migration}

An in-situ observation of interface migration is demonstrated in Figs. 7(a) - 7(d), in which the images were taken in a region close to a crack tip. The velocity of dislocation motion in a $\gamma / \gamma$ twin-related interface was found to be much faster than that shown in Fig. 5 because of a stress concentration caused by the crack. In fact, the dislocation velocity was so fast that it became difficult to track the motion of each dislocation in the dislocation array. The twin interface initially migrated with a high rate [Figs. 7(a) and 7(b), $v_{i} \sim 19 \mathrm{~nm} / \mathrm{sec}$ (1580 times faster than that in Fig. 4!)], and after 10 second the migration became much slower [Figs. 7(c) and 7(d), $v_{i} \sim 0.16$ $\mathrm{nm} / \mathrm{sec}]$. The interface subsequently migrated close to a $\gamma / \alpha_{2}$ interface. This rate change is presumably resulted from the interaction of stress field between $\gamma / \gamma$ and $\gamma / \alpha_{2}$ interfaces. Coarsening of the $\gamma$ lamella occurred as a result of the migration of $\gamma / \gamma$ interface.

\section{Summary}

Deformation-induced interface sliding and migration in lamellar TiAl have been directly observed using in-situ straining techniques performed in a transmission electron microscope. The results clearly show that the interfacial dislocations in lamellar interfaces are mobile even at ambient temperatures. Since step (ledge) is associated with the core of each interfacial dislocation, the lamellar interfaces can thus migrate through the cooperative motion of interfacial dislocations and resulting in the microstructural instability (coarsening/shrinkage) of constituent lamellae. Both the dislocation velocity and migration rate are very sensitive to the magnitude of resolved shear stress acting on the interfaces. The results of current study reveal that to appropriately control the mobility of interfacial dislocation is of paramount importance for the enhancement of both microstructural stability and creep resistance of refined lamellar TiAl especially at elevated temperatures. 

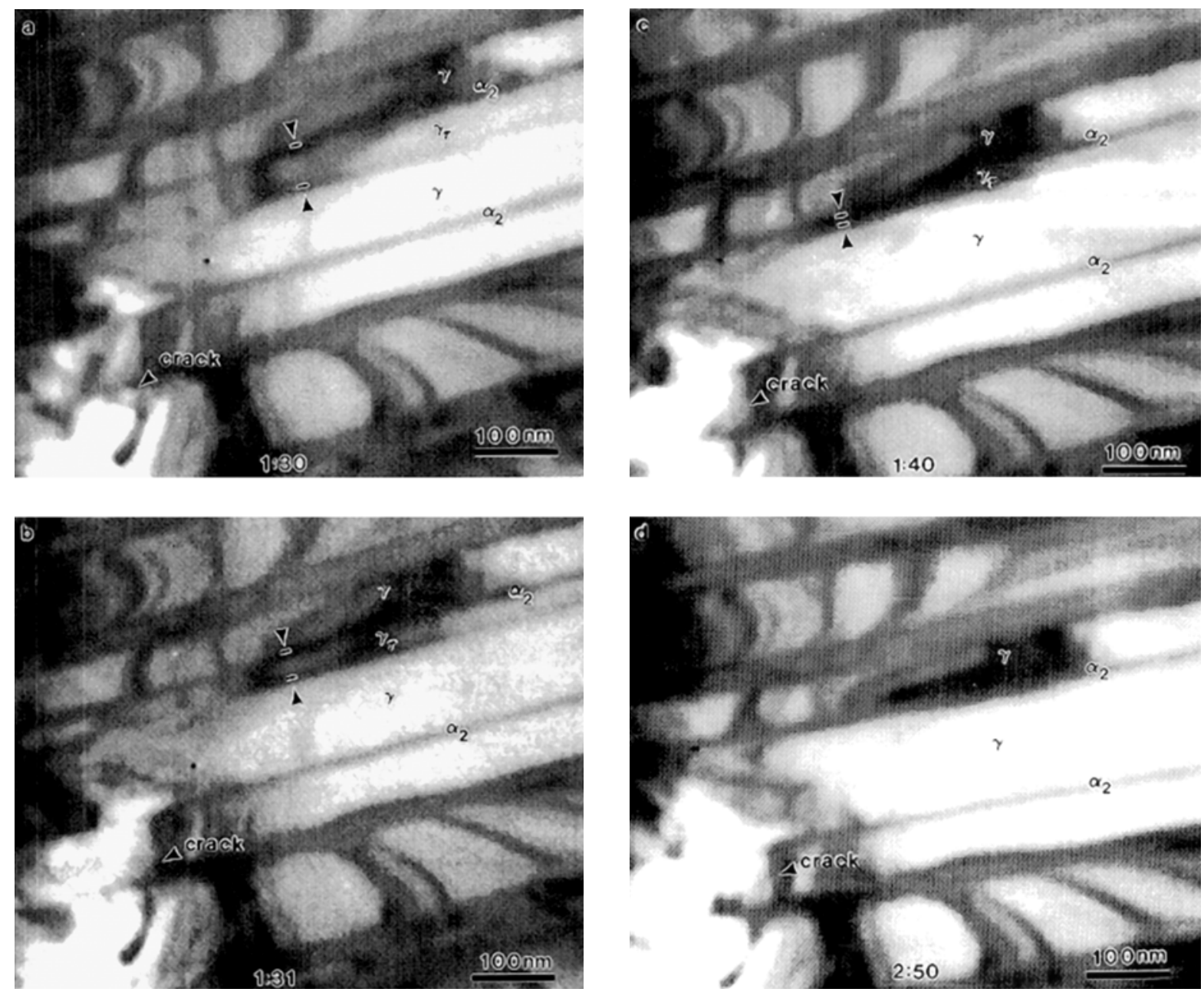

Fig. 6. Consecutive in-situ video images showing the migration of a twin-related interface near a crack tip; (a) $t=0,(b) t=1 s,(c) t=10 s,(d) t=80 s$.

Acknowledgements - This work was performed under the auspices of the U.S. Department of Energy by University of California, Lawrence Livermore National Laboratory under contract No. W-7405-Eng-48. The authors would like to thank Mr. M. A. Wall for his technical advices in carrying out the in situ straining experiment. The authors are also in debt to Dr. C. T. Liu of Oak Ridge National Laboratory for his technical guidance and providing the alloys used for this investigation.

\section{References}

1. L. M. Hsiung and T. G. Nieh, Intermetallics 7, 821 (1999).

2. L. M. Hsiung, T. G. Nieh, B.W. Choi, and J. Wadsworth, Mater. Sci. Eng., A329-331, (2002), 637.

3. L. M. Hsiung and T. G. Nieh, Mater. Sci. and Eng. (2003), in press.

4. M. Yamaguchi and Y. Umakoshi, Progress in Materials Science, 34, 1 (1990).

5. M. Yamaguchi and H. Inui, in Structural Internietallics, ed. R. Darolia et al., TMS, Warrendale, PA, 127(1993).

6. Y. Yamamoto, M. Takeyama, T. Matsuo, Mater. Sci. Eng., A329-331 (2002), 631.

7. L. Zhao and K. Tangri, Phil. Mag. A, 65 (1992), 1065.

8. G. J. Mahon and J. M. Howe, Metall. Trans. A, 21A (1990), 1655. 


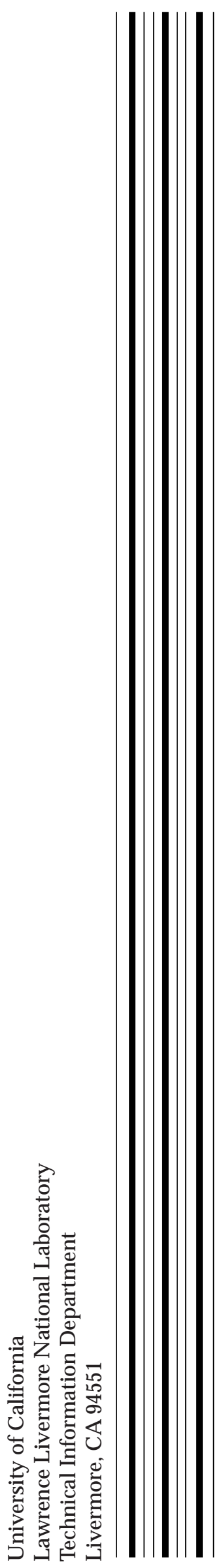

